\title{
A new path toward gravity experiments with anti-hydrogen
}

\author{
P. Perez, A. Rosowsky \\ DSM/Dapnia/SPP, CEA/Saclay, F-91191 GIF-SUR-YVETTE
}

\begin{abstract}
We propose to use a $13 \mathrm{KeV}$ antiproton beam passing through a dense cloud of positronium $(\mathrm{Ps})$ atoms to produce an $\overline{\mathrm{H}}^{+}$"beam". These ions can be slowed down and captured by a trap. The process involves two reactions with large cross sections under the same experimental conditions. These reactions are the interaction of $\bar{p}$ with $\mathrm{P}_{\mathrm{S}}$ to produce $\overline{\mathrm{H}}$ and the $e^{+}$capture by $\overline{\mathrm{H}}$ reacting on $\mathrm{P}_{\mathrm{S}}$ to produce $\overline{\mathrm{H}}^{+}$. Once decelerated with an electrostatic field and captured in a trap the $\overline{\mathrm{H}}^{+}$ions could be cooled and the $e^{+}$removed with a laser to perform a measurement of the gravitational acceleration of neutral antimatter in the gravity field of the Earth.
\end{abstract}

Key words: positron, positronium, matter antimatter symmetry, antigravity PACS: 41.75.Fr, 04.80.Cc

\section{Introduction}

The measurement of the gravitational mass of antiparticles motivates physicists since over three decades. It is interesting to note that general relativity would not contradict antiparticles to "fall up" in the gravity field of the earth [1]. Experiments to test such ideas have been proposed on positrons and antiprotons [2] but never succeeded. Several experiments at CERN are now producing neutral antimatter in the form of antihydrogen or antiprotonic helium atoms $[3,4]$ for CPT tests. The trapping of neutral antihydrogen atoms is the next step for these experiments. Some proposals to measure the fall of these atoms have been presented [5]. The possibility to measure the free fall of positronium has also been studied [6].

Email addresses: patrice.perez@cea.fr (P. Perez,), andre.rosowsky@cern.ch (A. Rosowsky). 


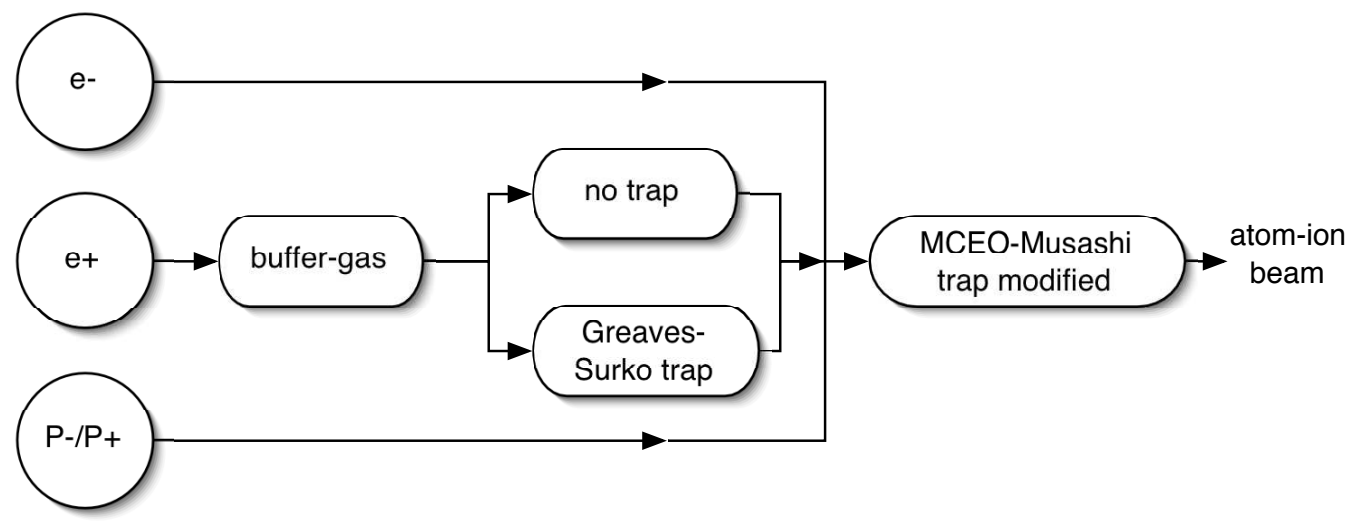

Fig. 1. Atoms/ions production scheme.

Recently it has been proposed [7,8] to measure the gravity acceleration of antimatter using the $\overline{\mathrm{H}}^{+}$ion. This ion has the advantage that it can be cooled down to $13 \mu \mathrm{K}$, a temperature suitable for a gravity experiment. The authors do not investigate in detail the $\overline{\mathrm{H}}^{+}$production scheme but suggest the interaction of $\mathrm{P}_{\mathrm{S}}$ Rydberg atoms with $\overline{\mathrm{H}}$ atoms $[9,10]$. In this article we concentrate on the $\overline{\mathrm{H}}^{+}$production scheme and propose a "beam to cloud" experimental configuration instead of a trapped system. This configuration allows the isolation of the $\overline{\mathrm{H}}^{+}$from the production region.

The positronium cloud hereafter referred to as "the target" is not contained: it is made of positronium atoms emitted from an Aluminium crystal surface which is bombarded by a flux of positrons. The atoms are emitted in a direction normal to the surface with a few mrd angular spread [11]. Their energy is $\sim 2 \mathrm{eV}$ and their speed is $\sim 1 \mathrm{~mm} / \mathrm{ns}$. The density of the Ps target is proportional to the positron flux. As explained further, an amount of order $10^{11}$ positrons is needed in order to get the required density for the Ps target. However, the short Ps lifetime requires this amount to be delivered in a few nanoseconds while the highest foreseen rates of slow positrons are at best $10^{11} \mathrm{~s}^{-1}$. The positrons have then to be accumulated in the crystal vicinity and accelerated toward the crystal. To counteract the effects of space charge, the positrons are accumulated into a small neutral $e^{-} e^{+}$"plasma" for a short time. The plasma size is $\sim 3 \mathrm{~mm}^{2} \times 1 \mathrm{~cm}$. Then an electrostatic field accelerates the positrons toward an Aluminium crystal where they are converted into Positronium atoms.

A $13 \mathrm{keV}$ antiproton beam is guided parallel to the crystal at a distance of $\sim 150 \mu \mathrm{m}$. The target length is crossed in $6 \mathrm{~ns}$ by the $13 \mathrm{keV}$ antiprotons.

The flux of positronium atoms is maintained as long as the flux of positrons toward the crystal is kept. It is the flux of positronium atoms which constitutes the "target". The overall layout for such a gravity experiment is presented in figure 1 . 
Aside from the gravity experiment, the technique to accumulate positrons in the plasma volume described in this article could prove useful as a first step toward the realization of a positronium [12] BEC, a $511 \mathrm{KeV}$ laser [13] and the observation of the antimatter molecule $\mathrm{Ps}_{2}$. When Greaves-Surko traps $[14,15,16]$ are available with a capability to hold $10^{12}$ positrons and provided that the neutral plasma can be held during the time required to empty the trap, one could produce a density of positronium of $\sim 0.310^{14} \mathrm{~cm}^{-3}$. An experiment to observe the stimulated annihilation process would then become feasible.

If traps with such capability are not available, an alternative path toward a stimulated annihilation observation could be to use the neutral plasma in a MCEO trap [17]. The positronium atoms produced by the 3 body reaction are in Rydberg states. It takes a few $\mu$ s before they reach the ground state. There, the magnetic field couples the triplet state to the singlet state, which has a lifetime of $0.125 \mathrm{~ns}$. Therefore the life time of the positronium produced inside the plasma is dominated by the decay time towards the ground state. Using an infrared laser onto the $\mathrm{P}_{\mathrm{s}}^{*}$ gas one can re-ionize some atoms while preventing the decay into states with a low level of excitation. Hence the life time can be increased and, in principle, longer trains of positrons could be filled into the plasma volume.

This configuration is also well suited for spectroscopy experiments where atoms containing a positron, or a positronium atom $[18,10]$ are produced by the interaction of atoms/ions with the target. The incoming antiproton beam is then replaced by an ion or atom beam.

The path described in this article to produce an antimatter ion beam is the following:

- the accumulation of positrons in a neutral $e^{-} e^{+}$plasma,

- the separation of $e^{-}$and $e^{+}$by an electrostatic field and the interaction of the $e^{+}$with an Aluminium crystal to produce a positronium cloud referred as the target,

- the interaction of $13 \mathrm{keV}$ (anti)protons with the target to produce the atoms and the ions as a beam.

The beam to cloud configuration presented in this article is made of several devices which were developped separately for various applications:

- the $10 \mathrm{MeV} e^{-}$beam on a thin foil to produce an intense $e^{+}$source,

- the buffer gas and Greaves-Surko trap,

- the MCEO trap,

- the Charge Focusing Aluminium Converter (CFAC).

The present study takes the parameters of these devices as they appear in 
the litterature and shows that with little modifications, these devices can be assembled to produce an $\bar{H}^{+}$ion beam.

The steps involved in the target production are discussed in the next section. The atom and ion production rates are presented in section 3. Several technical features are discussed in section 4.

\section{The positronium target}

The positronium target is obtained by accelerating positrons from an $e^{-} e^{+}$ plasma toward an Aluminium crystal. The positrons hit the crystal with a kinetic energy above $40 \mathrm{eV}$ to avoid elastic and specular reflection [19].

In the following we describe a way to produce the neutral plasma and the subsequent extraction and focalisation of the positrons from the plasma onto the Al crystal.

We foresee two modes of operation for the creation of the neutral plasma:

- the slow loading mode where the positrons are extracted continuously from the buffer gas section of the Greaves-Surko trap which cools them to room temperature $(\sim 25 \mathrm{meV})$. The loading time is a few seconds.

- the fast loading mode where the positrons are stored and cooled to $2 \mathrm{meV}$ in a Greaves-Surko trap [16] and extracted in $\sim 10 \mu \mathrm{s}$. This fast extraction heats the positron beam. This mode of operation requires less confinement time for the neutral plasma and may allow higher plasma densities.

The electrons are provided by a buffer gas or magnetic trap depending on the desired temperature. Since electrons do not annihilate on the container walls and are easy to produce, the final neutral plasma temperature is tuned by setting the electron beam temperature.

We assume that the positrons are produced continuously through the interaction of and intense beam of $10 \mathrm{MeV}$ electrons with a thin tungsten foil [20] with a rate, after solid Neon moderation, of $10^{11} \mathrm{~s}^{-1}$. The positrons are cooled to room temperature $(\sim 25 \mathrm{meV})$ in a buffer gas. In the slow loading mode the positrons are taken at the exit of the buffer gas section while in the fast loading mode they are first accumulated in a Greaves-Surko trap $[16,14,15]$ where they are stored and cooled to $\sim 2 \mathrm{meV}$. The low temperature of the positrons is a feature of the Greaves-Surko trap: the strong magnetic field is produced by a supraconducting magnet and the positrons are in thermal equilibrium with the magnet container at a temperature of $2 \mathrm{meV}$. Such a low temperature enhances the 3 body reaction which absorbs the positrons if the 

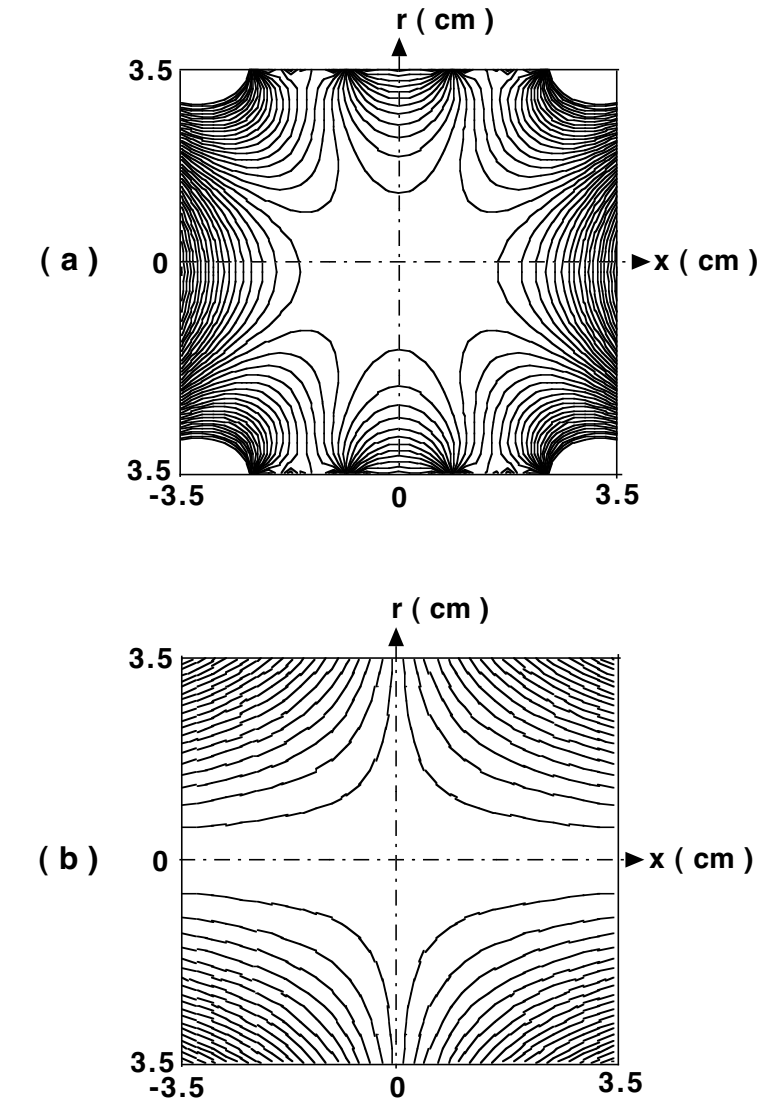

Fig. 2. The MCEO trap electric potential (a) and magnetic flux (b) set for antihydrogen synthesis [17].

neutral plasma were to be stored in this trap.

The preferred device for accumulating the $e^{+}$in a neutral plasma is the MCEO trap [17] which does not require a very low temperature such as the one reached in a Greaves-Surko trap. Therefore the heating of the positrons can be accomodated and is beneficial to accumulate enough positrons in the neutral plasma.

The positrons are guided by a 100 Gauss magnetic field, from the GreavesSurko trap (or the buffer gas), to the neutral plasma. The injection pipe is shielded to minimize its leaking field.

The original MCEO trap is a magnetic cusp with an octupolar electric field. It is made of several pairs of coils of $10 \mathrm{~cm}$ radius arranged symmetrically with respect to the center of the trap. The most central coils are located at 4 $\mathrm{cm}$ from the center. The other coils are separated from each other by $5 \mathrm{~cm}$. The currents in each pair of symmetric coils are of opposite sign. The current 
amplitude can be varied for each pair.

It is designed to mix both positrons and antiprotons. It is to be implemented in the ASACUSA beam line at CERN under the name of Musashi trap. In our application this trap is meant to mix positrons with electrons. Therefore a scaled down version has to be designed.

The scaled down version of the MCEO trap has the following parameters: the magnetic coils radius is $1.5 \mathrm{~cm}$, the most central coils are at $1.2 \mathrm{~cm}$ from the center, the next coils are separated by $1.5 \mathrm{~cm}$, the total current for each set of coils is $4 \mathrm{k}$ Ampere-turns, the octupolar electrostatic field is set by a central electrode at $70 \mathrm{~V}$ and a length of $1.0 \mathrm{~cm}$. The key feature of this trap is to have a null field region in its center (figure 2). Once a charged plasma is loaded, it acts as a potential well for the opposite charge. We use electrons to create the well for the positrons. The neutral plasma will be located at the center of the well. The $e^{-}$and $e^{+}$beams are injected along the $\mathrm{x}$ axis. The octupolar electrostatic field of the MCEO trap is simulated using an analytical form [21].

A simple trap made of two coils acting as mirrors [22] is also considered but the injection of the positrons is more difficult.

In order to extract the positrons from the neutral plasma and accelerate them toward the crystal, an electrostatic field is used. The electrode configuration is shown in figure 3 .

The electrodes are arranged in two parallel planes. The cathode is made of Al cristal. Its surface is $100 \mu \mathrm{m} \times 1 \mathrm{~cm}$. The neutral plasma is located in the volume between the two planes. This configuration will be referred as Charge Focusing Aluminium Converter or CFAC.

This configuration is inspired by a technology developped for high energy physics detectors called the Micro Strip Gas Chamber [23], or MSGC. The distances between electrodes and field magnitude are taken from this development.

In the CFAC, the electrostatic field of the MSGC is modified by introducing 4 wires to smoothe the side field as shown in figure 4 .

The CFAC was put in the center of the trap by the simulation described below in order to get an estimation of the positronium density and time distribution. The insertion of metallic conductors in the center of the MCEO trap is unrealistic. An integrated design is proposed in the discussion section.

A fortran program was written to simulate:

- the stability of the neutral plasma during the $10 \mu$ s loading time, 


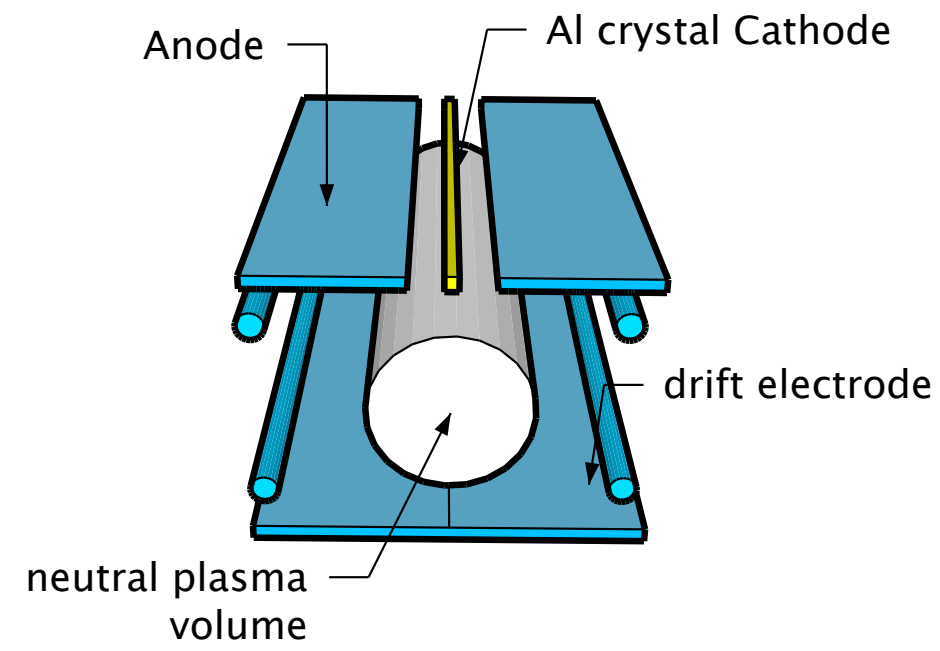

Fig. 3. The CFAC electrodes geometry.

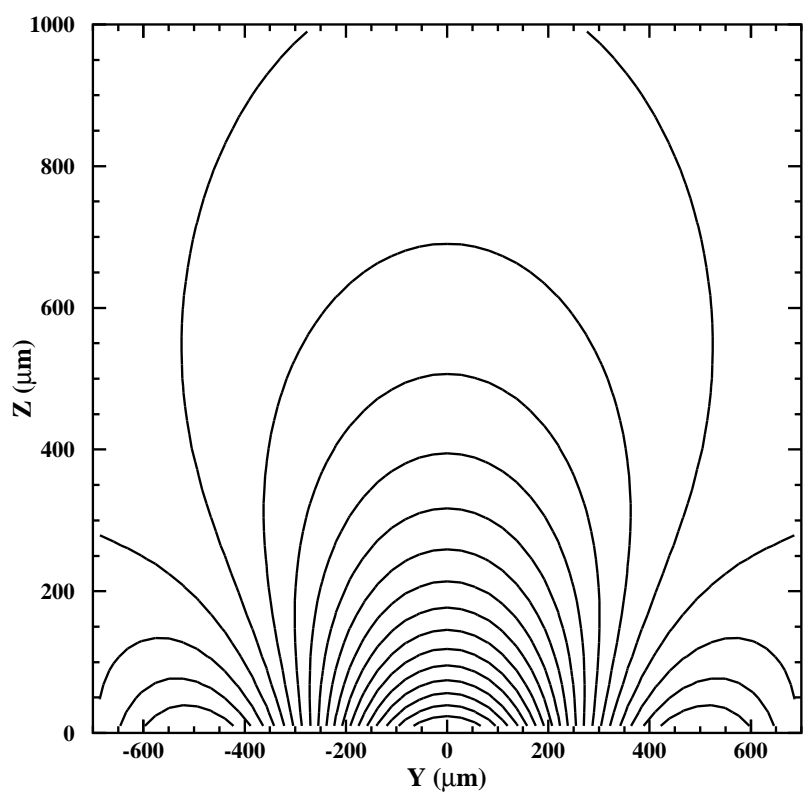

Fig. 4. The CFAC electrostatic potential: $(0,0)$ is the center of the crystal surface.

- the acceleration by the CFAC electrostatic field and the time distribution of the positrons hitting the Al crystal. 


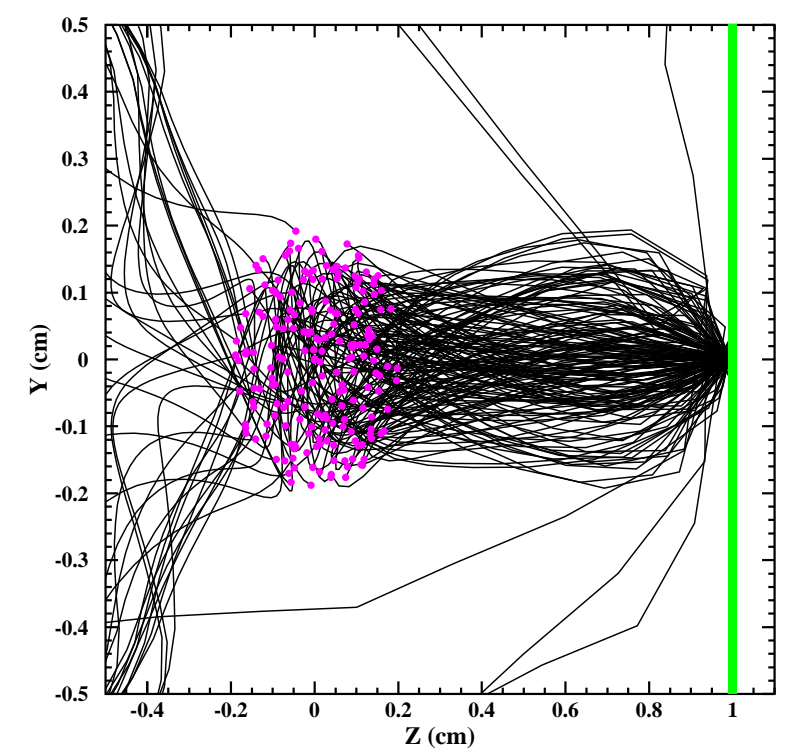

Fig. 5. Positron tracks from the plasma to the CFAC in $\mathrm{cm}$.

\subsection{The target simulation}

In the MCEO trap the positrons are injected along its axis, parallel to the magnetic field which guides them.

\subsubsection{The CFAC electrostatic field}

The CFAC electrostatic field is computed using analytical formulae [23]. The gap of the CFAC, i.e. distance between the drift electrode and the cathode planes is $1.5 \mathrm{~cm}$. The cathode plane is located at $1 \mathrm{~cm}$ from the $\mathrm{x}$ axis and parallel to it. The drift electrode size is $1 \mathrm{~cm} \mathrm{x} 7 \mathrm{~mm}$. The cathode, made of the $\mathrm{Al}(111)$ crystal has a $100 \mu \mathrm{m}$ width. The distance between the edge of the cathode and the next electrode in the cathode plane is $200 \mu \mathrm{m}$. The drift electrode plane being at $1600 \mathrm{~V}$, the cathode is grounded and the bias voltage is $1550 \mathrm{~V}$. The plasma temperature is $30 \mathrm{meV}$.

During the loading phase the CFAC field is set "off". The acceleration phase starts when the CFAC electrostatic field is set "on" while the octuplole electrostatic field and the cusp magnetic field are set "off".

The positron capture and the emission of positronium atoms is a fast process, the delay of which can be neglected at the nanosecond scale [24]. Therefore the time distribution of the positrons hitting the crystal is also the time distribution of the positronium atoms exiting the crystal surface (figures 5,6). 


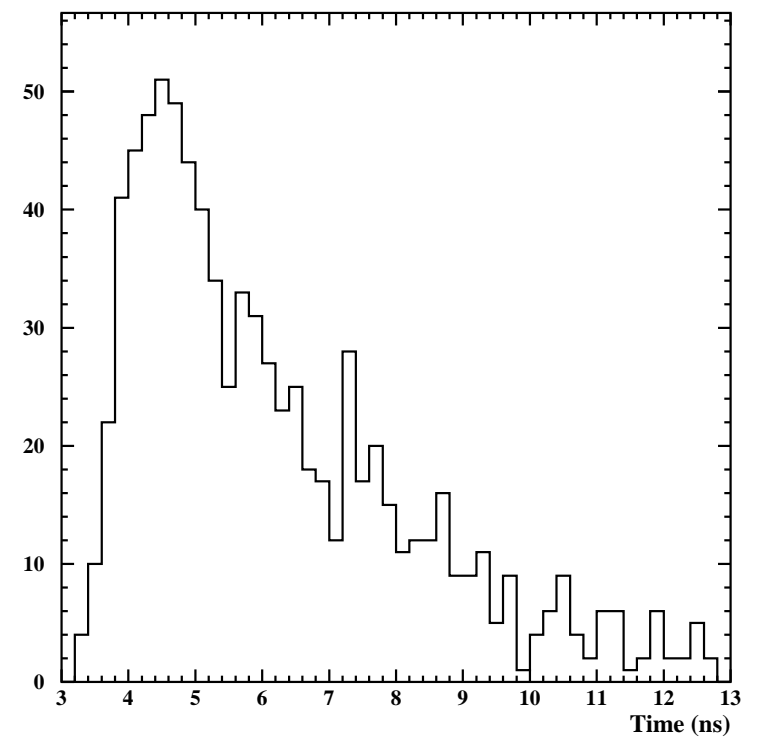

Fig. 6. Arrival time distribution of the positrons on the crystal in nanoseconds.

The positronium atoms exiting the crystal are in the ground state. The magnetic field couples the singlet and triplet states [25], reducing the Positronium life time to a minimum of 0.125 ns.

In an experimental test with a MCEO trap and with electrons the radial field on the central electrode surface was 360 Gauss [26]. This trap is designed to hold both positrons and antiprotons. When antiprotons are used the magnetic field is much stronger but in the beam to cloud configuration the antiprotons cross the trap axis and are not kept. Hence with a weak field ( 3.5 Gauss) we take as effective lifetime 0.200 ns. This life time corresponds to a travel distance of $200 \mu \mathrm{m}$. The target volume is defined as a box parallel to the crystal, which starts at a distance of $100 \mu \mathrm{m}$. The target is $100 \mu \mathrm{m}$ large, $1 \mathrm{~cm}$ long and $100 \mu \mathrm{m}$ thick, which gives a volume of $10^{-4} \mathrm{~cm}^{3}$. This target volume is centered at a distance of $150 \mu \mathrm{m}$ from the crystal surface.

An estimate of the target density is obtained by assuming all Ps atoms to fly perpendicularly from the crystal surface [11]. The number of positrons stored in the plasma is $N_{e^{+}}=10^{11}$. The positron flux from the plasma to the crystal has a duration of $\sim 10 \mathrm{~ns}$. The Ps atoms spend only $0.100 \mathrm{~ns}$ in the target volume, hence the target density is given by:

$$
n_{P_{s}}=\frac{10^{11}}{10^{-4}} \frac{0.100}{10} \epsilon_{P_{s}} \epsilon_{C F A C}=0.2510^{13} \mathrm{~cm}^{-3}
$$

The target volume could also be counted starting from the crystal surface with some antiproton losses (antiprotons hitting the crystal). 
The simulation of the CFAC gives an efficiency of $\epsilon_{C F A C}=0.5$ for a positron to reach the crystal. The positronium emission efficiency from the crystal surface is taken as $\epsilon_{P_{s}}=0.5$.

A correcting factor due to the positronium decay between the crystal and the target volume will reduce the density by less than a factor 2 . This correction depends on the magnetic field strength.

\subsection{Positron losses}

When mixing $e^{-}$and $e^{+}$with a density above $\sim 10^{9} \mathrm{~cm}^{-3}$ and a temperature of a few meV, the plasma collapses into positronium through a 3 body interaction.

$$
e^{-}+e^{+}+e^{ \pm} \longrightarrow \mathrm{P}_{\mathrm{S}}\left(\mathrm{n}^{\prime} \mathrm{l}^{\prime}\right)+e^{ \pm}
$$

The rate of other reactions, namely the radiative recombination and the direct annihilation are negligible. This reaction and the subsequent positronium annihilation destroy the positrons.

The number of positrons in the neutral plasma as a function of time is given by ${ }^{1}$ :

$$
\begin{array}{r}
N_{e}=\sqrt{\frac{a}{\lambda}} \times \tanh (\sqrt{a \lambda} t) \\
\lim _{t \rightarrow \infty} N_{e}(t)=\sqrt{\frac{a}{\lambda}}
\end{array}
$$

Where $a$ is the injection rate and $\lambda$ is a constant related to the 3 body reaction.

The tables obtained in the appendix show that in the worst case, in $\sim 2 \mathrm{~s}$ and at a temperature equal or above $4 \mathrm{meV}$ it is possible to accumulate $210^{11} e^{+}$in the plasma. For less than 10 seconds of accumulation there is little variation with temperature above $4 \mathrm{meV}$. Once the injection of positrons ends and during $\sim 1$ s the loss of positrons is small and on the time scale of a few $\mu$ s it can be neglected.

In order to have a negligible loss by annihilation on remaining gas a vacuum environment is required with a residual gas partial pressure below $\sim 10^{-9}$ torr.

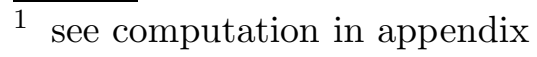




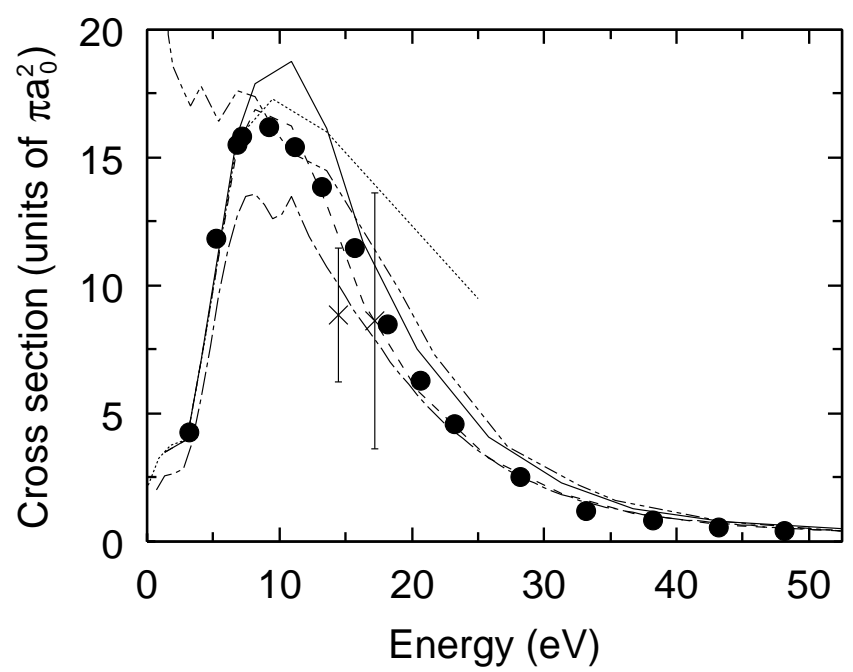

Fig. 7. Hydrogen formation cross section in $\mathrm{P}_{\mathrm{S}}+\mathrm{p} \rightarrow \mathrm{H}+e^{+}$collisions [27]. Closed circles represent the calculation of Yamanaka and Kino; lines, other models referenced in the same paper; crosses, the experiment of Merrison et al. $[28,31]$. The abscissa is the energy of the proton in the center of mass frame.

\section{Antihydrogen production}

The charge exchange on positronium to produce the atom is a resonant reaction. The cross-section calculations $[27,29,30]$ have been confirmed by an experiment $[28,31]$.

Once the $\overline{\mathrm{H}}$ atom is produced, it captures a positron to make the ion. There is a non resonant channel, the two body recombination, and a resonant one, the charge exchange on positronium.

The choice of the antiproton kinetic energy is given by the overlap of the resonant cross sections. In the region of interest, the antiprotons have a kinetic energy of $\sim 13 \mathrm{keV}$ (figure 7 ). The atom production cross section obtained experimentally [28] is $7.8 \pi a_{0}^{2}{ }^{2}$. At this kinetic energy, the computed cross section [32] for the ion production is $\sim 0.05 \pi a_{0}^{2}$ (figure 8 ).

The (anti)hydrogen production rate normalized to the (anti)proton flux is:

$$
n_{P_{s}} \times \sigma_{\overline{\mathrm{H}}} \times v_{\bar{p}}
$$

The (anti)proton speed at $13 \mathrm{keV}$ is $v_{P}=1.5810^{8} \mathrm{cms}^{-1}$. Therefore the normalized rate is $0.2810^{6} \mathrm{~s}^{-1}$. The target length is $1 \mathrm{~cm}$, hence the crossing time

$\overline{2 \text { Here }} a_{0}$ is the Bohr radius and $\pi a_{0}^{2}=0.880 \times 10^{-16} \mathrm{~cm}^{2}$. 


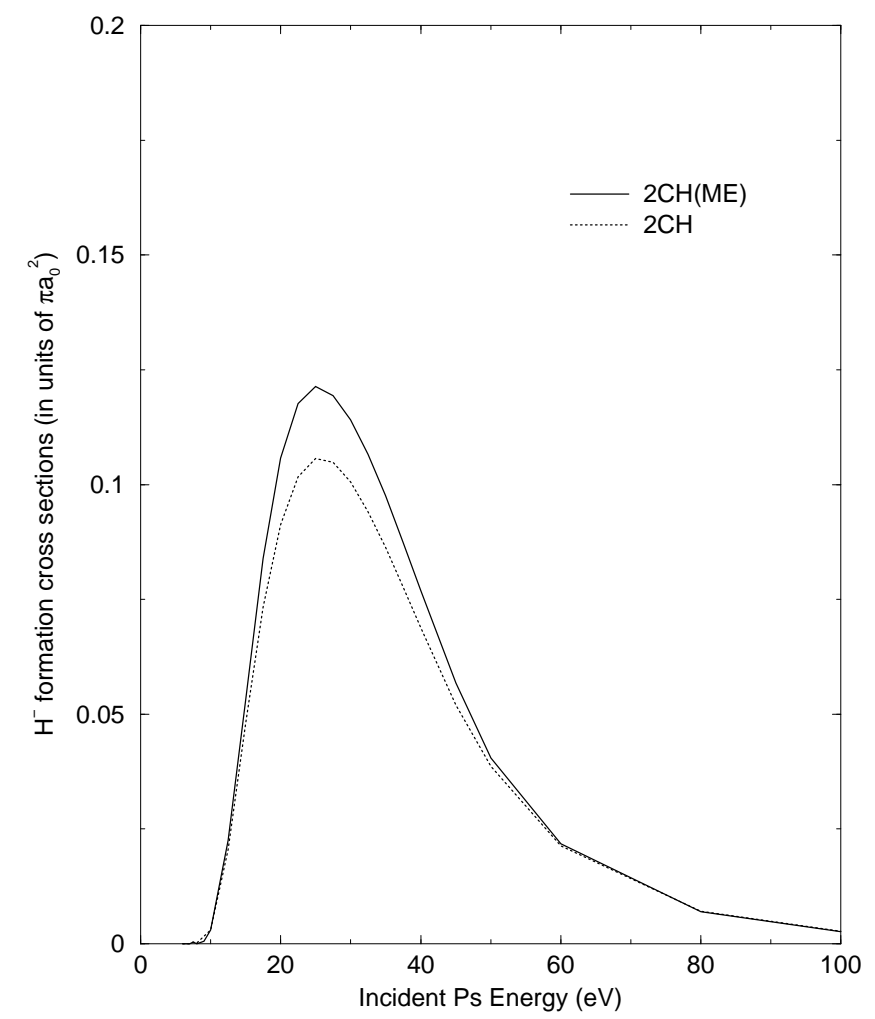

Fig. 8. $\mathrm{H}^{-}$production cross section in $\mathrm{H}(n=1)+\mathrm{P}_{\mathrm{S}}\left(n^{\prime}=1\right) \rightarrow \mathrm{H}^{-}+e^{+}$collisions [32]. The lines are two variants of a two-channel calculation by Biswas. The abscissa is the energy of the Ps in the center of mass frame.

by the (anti)proton beam is $6.3 \mathrm{~ns}$ and the number of antihydrogen atoms produced by each antiproton crossing the target is 0.0017 .

The (anti)hydrogen ion production rate normalized to the antihydrogen flux is:

$$
n_{P_{s}} \times \sigma_{\overline{\mathrm{H}}^{+}} \times v_{\overline{\mathrm{H}}}
$$

The normalized rate is $0.1810^{4} \mathrm{~s}^{-1}$. After convolution for the $\bar{H}$ production in the same $1 \mathrm{~cm}$ long target, the number of ions produced for each antiproton crossing the target is $10^{-7}$.

At the edge of the crystal and at a distance of $150 \mu \mathrm{m}$ the CFAC electrostatic field component transverse to the antiproton path is $\sim 0.12$ Mega Volts $/ m$. Therefore an antiproton aiming at the edge of the crystal will be pulled transversally by $240 \mu \mathrm{m}$ on a distance of $1 \mathrm{~cm}$. The transversal electrostatic field which is null on the axis and grows toward the edges of the target, will cause a maximum pull $\sim 0.5 \mathrm{~mm}$ and a beam divergence of $\sim 50 \mathrm{mrd}$. 


\section{Discussion}

In the integration of the devices (CFAC, MCEO trap, buffer-gas, GreavesSurko trap) into a single experiment there is room for changing their parameters: gains from tuning the device parameters and losses are difficult to simulate and have to be established experimentally. When changing the parameters in the simulation these gains/losses are within a factor 10 .

Still there are many technical questions which are not addressed by the simulation. We shall now list some of them.

The field in the buffer gas trap is 1.5 kGauss and $5 \mathrm{~T}$ in the Greaves-Surko trap: a set of iron plates and auxilliary fields shall be designed to reach the 100 Gauss guiding solenoidal field [16].

Positron plasmas with a density of $10^{10} e^{+} \mathrm{cm}^{-3}$ at meV temperatures have been stored in a Greaves-Surko trap with a few $10^{8}$ trapped $e^{+}$. The next generation of multicell traps $[16,14,15]$ is expected to store and cool to meV temperature $10^{13} e^{+}$. The fast loading mode cannot be implemented without traps that can store at least $10^{11} e^{+}$. In this fast mode, once trapped, the positrons shall be extracted in a single pulse. This step has to be experimentally established.

Such traps cannot be filled with $e^{+}$emitted by a radioactive source in less than a few weeks. An intense source of slow positrons such as the one we proposed [20], using an intense $10 \mathrm{MeV}$ electron beam impinging at a small incidence angle on a thin tungsten foil is expected to fill the trap at a rate of $10^{10} e^{+} \mathrm{s}^{-1}$.

In the slow loading mode, no trap is needed and only a buffer gas is required.

At high density, the neutral plasma is not transparent to the electro-magnetic field. The extraction of the positrons by the CFAC field in $\sim 10$ ns requires a pulse shaping technique [33] and shall be tested experimentally.

In order to implement a gravitation experiment with a scheme such as the one discussed by J. Walz and T. W. Hänsch [7] constraints have to be met by the antiproton source. The ions are produced in a beam at $13 \mathrm{KeV}$ with an energy and angular spread dominated by the initial (anti)proton energy spread and the $\sim 50$ mrd divergence due to the CFAC field. The angular spread due to the charge exchange reactions is negligible. The angular spread shall be taken into account to decelerate and collect the $\overline{\mathrm{H}}^{+}$into an ion trap with high efficiency [34]. Antiproton traps already exist, but a trap with $10^{6} \overline{\mathrm{p}}$ capable of producing a beam of $13 \mathrm{keV} \pm 1 \mathrm{keV}$ energy with a divergence lower than $0.01 \mathrm{rd}$ (ie $100 \mu \mathrm{m}$ on $1 \mathrm{~cm}$ path) is still to be demonstrated. 


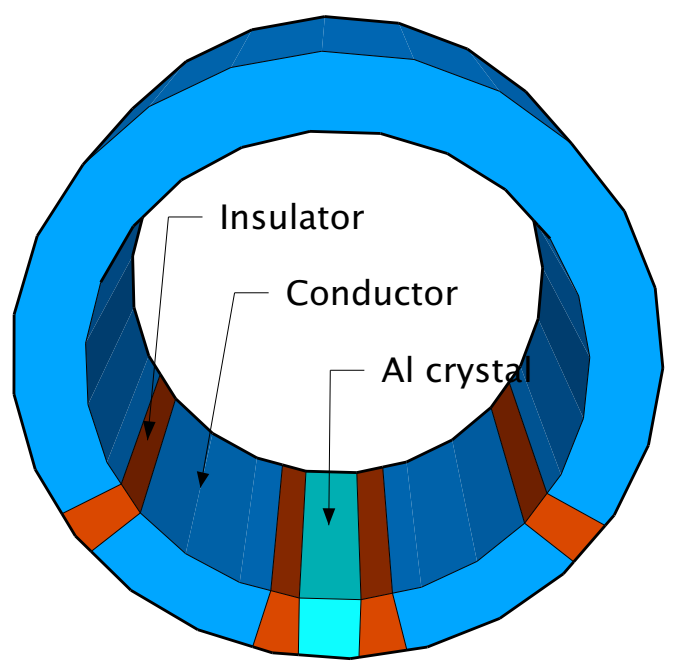

Fig. 9. Modified central electrode of a MCEO trap to produce an approximate CFAC field.

For antihydrogen creation, the very large $e^{ \pm}$plasma densities discussed above allow to transform the antiprotons with a relatively high efficiency. But antiprotons are difficult to produce. However, in order to test the process one can use protons and an existing trap: the lower plasma density being compensated by a larger number of protons (say $10^{8} \mathrm{p}$ per beam pulse).

Another aspect of this path for a gravity experiment is that the reactions involved are at all steps charge symmetric: by switching from protons to antiprotons on the time scale of 1 hour, it is possible to measure $g$ in the same gravitational field with little or no tide effect due the movement of the moon and other masses.

The integration of the CFAC into a MCEO trap requires some modification. We foresee to cut the central electrode in 4 (or more) sections separated by a thin insulator (figure 9). In the trapping mode all the sections are set at the same potential acting as a single electrode. In the CFAC mode the potentials are set to produce an electrical field similar to the one in figure 3 .

The $\overline{\mathrm{H}}^{+}$ion beam is needed for the gravity experiment, but a large number of $\overline{\mathrm{H}}$ atoms are also produced in the beam. Using a laser to stimulate the transition toward the $n=2$ level one gets a beam in the $\sim 10-20 \mathrm{keV}$ range. Then the Separated Oscillatory Field method $[35,36,37]$ allows to measure the $2 S_{1 / 2}-2 P_{3 / 2}$ fine structure.

\section{Appendix}


The technique described in this article can be used for experiments on positronium BEC and a $511 \mathrm{keV}$ laser because it could reach a positronium density of $\sim 10^{14}$ or $\sim 10^{15} \mathrm{~cm}^{-3}$. The appendix gives a numerical estimation of the density as a function of time and temperature.

The maximum number of positrons available to interact with an Aluminium crystal is the result of the equilibrium between the injection rate and the 3 body reaction rate. This reaction (see equation 2 ) and the subsequent positronium annihilation destroy the positrons.

The time evolution of the number of positrons in the plasma is given by the injection rate and the 3 body reaction rate [38]: $d N_{e}=a d t-r_{3} d t$

where $N_{e}$ is the number of $e^{+}$in the plasma volume, $a$ is the injection rate and $r_{3}$ is the 3 body reaction rate given by: $r_{3}=A \times n_{e}^{2} \times v_{e} \times b^{5}$

where the electron thermal velocity $v_{e}=\sqrt{\frac{k T}{m_{e}}}$ and the impact parameter $b=\frac{e^{2}}{k T}$ are numerically: $v_{e}\left(\mathrm{~cm} \cdot \mathrm{s}^{-1}\right)=4.1910^{7} \sqrt{T(\mathrm{eV})}$ and $b\left(\mathrm{~cm} \cdot \mathrm{eV}^{-1}\right)=$ $1.4410^{-7} / T(\mathrm{eV})$, resulting in $r_{3}\left(\mathrm{~s}^{-1}\right)=2.610^{-27} A \times n_{e}^{2} / T^{4.5}$

where $A$ is a parameter which varies with the magnetic field, $n_{e}$ is the density in $\mathrm{cm}^{-3}$ and $T$ is the plasma temperature in $\mathrm{eV}$.

The neutral plasma volume is $\sim 30 \mathrm{~mm}^{3}$.

Let $V$ be the plasma volume and let's define $\lambda$ as $\lambda=2.610^{-27} A /\left(V^{2} T^{4.5}\right)$, so that $r_{3}=\lambda N_{e}^{2}$.

With the initial condition $N_{e}(t=0)=0$, we get: $d N_{e}=\left(a-\lambda N_{e}^{2}\right) d t$

Which leads to: $N_{e}=\sqrt{\frac{a}{\lambda}} \times \tanh (\sqrt{a \lambda} t), \lim _{t \rightarrow \infty} N_{e}(t)=\sqrt{\frac{a}{\lambda}}$

For the two extreme situations, $B=0$ and $B=\infty$, the $A$ parameter is respectively equal to 0.76 and 0.07 .

In the computations the ion was supposed to be much heavier than the electron and therefore its trajectory in the field was neglected [38]. Recently a computation was made with a proton taking into account its trajectory [39] for fields of a few Tesla: the variation of the parameter $A$ compared to the infinite field value was less than a factor 2 . Here the ion is the positron which is much lighter and the magnetic field is a few Gauss only. Therefore the positron behaves as a heavier ion in a stronger field. The expected behavior is then an intermediate one, between the above computation with 0 field and with infinite field.

A computation involving only electrons and positrons predicts a three body 


\begin{tabular}{|c|cccccc|}
\hline $\mathrm{T}(\mathrm{meV})$ & 2 & 4 & 10 & 25 & 50 & 100 \\
\hline $0.1 \mathrm{~s}$ & $9.9910^{9}$ & $9.9910^{9}$ & $9.9910^{9}$ & $9.9910^{9}$ & $9.9910^{9}$ & $1.010^{10}$ \\
$1 \mathrm{~s}$ & $9.0910^{10}$ & $9.9510^{10}$ & $9.9910^{10}$ & $9.9910^{10}$ & $9.9910^{10}$ & $1.010^{11}$ \\
$2 \mathrm{~s}$ & $1.4510^{11}$ & $1.9610^{11}$ & $1.9910^{11}$ & $1.9910^{11}$ & $1.9910^{11}$ & $2.010^{11}$ \\
\hline
\end{tabular}

Table 1

Number of $e^{+}$accumulated in the $30 \mathrm{~mm}^{3}$ neutral plasma with an injection rate $a=10^{11} s^{-1}$ for $\mathrm{A}=0.76(\mathrm{~B}=0)$

Table 2

\begin{tabular}{|c|cccccc|}
\hline $\mathrm{T}(\mathrm{meV})$ & 2 & 4 & 10 & 25 & 50 & 100 \\
\hline $0.1 \mathrm{~s}$ & $9.9910^{9}$ & $9.9910^{9}$ & $9.9910^{9}$ & $9.9910^{9}$ & $1.010^{10}$ & $1.010^{10}$ \\
$1 \mathrm{~s}$ & $9.9110^{10}$ & $1.010^{11}$ & $1.010^{11}$ & $1.010^{11}$ & $1.010^{11}$ & $1.010^{11}$ \\
$2 \mathrm{~s}$ & $1.9310^{11}$ & $2.010^{11}$ & $2.010^{11}$ & $2.010^{11}$ & $2.010^{11}$ & $2.010^{11}$ \\
\hline
\end{tabular}

Number of $e^{+}$, accumulated in the $30 \mathrm{~mm}^{3}$ neutral plasma with an injection rate $a=10^{11} s^{-1}$ for $\mathrm{A}=0.07(\mathrm{~B}=\infty)$

\begin{tabular}{|c|cccccc|}
\hline $\mathrm{T}(\mathrm{meV})$ & 2 & 4 & 10 & 25 & 50 & 100 \\
\hline $\mathrm{A}=0.76$ & $1.8110^{11}$ & $8.6010^{11}$ & $6.7610^{12}$ & $5.3110^{13}$ & $2.5310^{14}$ & $1.2010^{15}$ \\
$\mathrm{~A}=0.07$ & $5.9510^{11}$ & $2.8310^{12}$ & $2.2310^{13}$ & $1.7510^{14}$ & $8.3210^{14}$ & $3.9610^{15}$ \\
\hline
\end{tabular}

Table 3

$\sqrt{\frac{a}{\lambda}}=$ maximum number of $e^{+}$, that can be accumulated in the $30 \mathrm{~mm}^{3}$ neutral plasma with an injection rate $a=10^{11} s^{-1}$ for $\mathrm{A}=0.76(\mathrm{~B}=0)$ and $\mathrm{A}=0.07$ (B $=\infty$ )

recombination rate twice greater than for protons and electrons [40].

The computed number of positrons accumulated inside the neutral plasma for several temperatures is tabulated for both values of $A$.

When the injection in the plasma ends, the evolution is given by the rate of the 3 body reaction:

$d N_{e}=-r_{3} d t=-\lambda N_{e}^{2} d t$

$N_{e}=\frac{N_{0}}{1+N_{0} \lambda t}$

\section{Acknowledgements}

We wish to express our sincere thanks to all the people from different fields with whom we had fruitful discussions: P.K.Biswas, G. Chardin, C. Guyot, B. Mansoulié, C. Surko, R. Greaves, A.P. Mills, J.M. Rax, Y. Yamazaki.

One of us is grateful to $\mathrm{Chi}-\mathrm{Yu} \mathrm{Hu}$ [29] who carefully presented the use of positronium to produce antihydrogen at the poster session of the ICPEAC 2001 conference. 


\section{References}

[1] B. Carter, Phys. Rev. 174 (1968) 1559; M.M. Nieto and T. Goldman, Phys. Rep. 205(5) (1991) 221 and Phys. Rep. 216 (1992) 343 E; G. Chardin, Quantum limits to the second law, San Diego (USA), Eds. AIP, Proc. 643 (2002) 385; G. Chardin, J.M. Rax, Phys. Lett. B282 (1992) 256; G. Chardin, Nucl. Phys. A558 (1993) 447; M. Charlton, Phys. Rep. 241 (1994) 65.

[2] F.C. Witteborn and W.M. Fairbank, Nature 220 (1968) 436; F.C. Witteborn and W.M. Fairbank, Phys. Rev. Lett. 19 (1967) 1049; J.M. Lockhart, F.C. Witteborn and W.M. Fairbank, Phys. Rev. Lett. 67 (1991) 283; N. Beverini et al., CERN/PSCC/86-26 and LANL Report LA-UR-86-260; V. Bouchiat, G. Chardin, M.H. Devoret and D. Esteve, Hyperfine Interactions109 (1997) 345.

[3] M. Amoretti et al., Nature, 419 (2002) 456; M. Amoretti et al., Phys. Lett. B578 (2004) 23-32.

G. Gabrielse et al., Phys. Lett. B548 (2002) 140; Phys. Rev. Lett. 89, 233401 (2002); Phys. Rev. Lett. 89, 213401 (2002).

H. Yamaguchi et al., Phys. Rev. A70 (2004) 012501.

[4] G. Gabrielse, Atom Made Entirely of Antimatter: Two Methods Produce Slow Antihydrogen, to appear in Advances in Atomic, Molecular and Optical Physics 50 August 2004.

[5] C. L. Cesar, Hyp. Interact. 109 (1997) 293-304; T. J. Phillips, Hyp. Interact. 109 (1997) 357-365.

[6] A. P. Mills Jr. and M. Leventhal, Nucl. Inst. Meth. B192 (2002) 102.

[7] J. Walz and T. Hänsch, General Relativity and Gravitation 36 (2004) 561

[8] J. Waltz, H. Pittner, M. Hermann, P. Fendel, B. Henrich, T. W. Hänsch, Cold antihydrogen atoms, Appl. Phys. B77, 713-717 (2003).

[9] M. Charlton, Phys. Lett. A143 (1990) 143.

[10] R.G. Greaves and C. Surko, Phys. Plasma 4 (5) May 1997, p1528-1543.

[11] D. M. Chen, S. Berko, K. F. Canter, K. G. Lynn, A. P. Mills Jr, L. O. Roellig, P. Sferlazzo, M. Weinert, R. N. West, Phys. Rev. Lett. 58 No 9 (1987) 921-4; K. G. Lynn, A. P. Mills Jr, R. N. West, S. Berko, K. F. Canter, L. O. Roellig, Phys. Rev. Lett. 54 (1985) 1702-5.

[12] P.M. Platzman and A.P. Mills Jr., Phys. Rev. B49 (1994) 454.

[13] A.P. Mills, Nucl. Inst. Meth. B192 (2002) 107.

[14] T.J. Murphy and C.M. Surko, Phys. Rev. A46 (1992) 5696; C.M. Surko, S.J. Gilbert and R.G. Greaves, Non-Neutral Plasma Phys. III, edited by J.J. Bollinger, R.L. Spencer and R.C. Davidson, (American Institute of Physics, New York), 3-12 (1999); http://physics.ucsd.edu/research/surkogroup/positron/buffergas.html 
[15] R. Greaves et C.M. Surko, Nucl. Inst. Meth. B192 (2002) 90; Radiation Physics and Chemistry 68, (2002) 419-25; Phys. Rev. Lett. 85, (2000) 1883-6; Canadian Journal of Physics 74, (1996) 445-8.

[16] R.G. Greaves and C.M. Surko, Practical limits on positron accumulation and the creation of electron-positron plasmas, Non-Neutral Plasma Physics IV, edited by F. Anderegg et al. (American Institute of Physics),0-7354-0050-4/02, (2002) 10.

[17] A. Mohri and Y. Yamazaki, Europhys. Lett., 63 (2003) 207-213; A. Mohri, T. Yuyama, H. Tanaka, H. Higaki, Y. Yamazawa and T. Michishita, Japan. J. Appl. Phys. 39 pp6726-6731 (2000); A. Mohri, T. Yuyama, H. Tanaka, H. Higaki, Y. Yamazawa, M. Aoyagi and T. Michista, Japan. J. Appl. Phys. 37 pp664-670 (1998); A. Mohri, T. Yuyama, Y. Kiwamoto, Y. Yamazawa and T. Michista, Japan. J. Appl. Phys. 37 L1553-L1555 (1998); A. Mohri and Y. Yamazaki, Europhys. Lett. 63 (2) pp207-213 (2003).

[18] D. Schrader in "Principles and Applications of Positron and Positronium Chemistry", Y. C. Jean et al.(2003) 26-32.

[19] A. P. Mills Jr and P. M. Platzman, Solid State Communications, 35 321-4.

[20] P. Perez and A. Rosowsky, Nucl. Inst. Methods A532 (2004) 523-532.

[21] T.N. Tiouririne, L. Turner and A.W.C. Lau, Phys. Rev. Lett. 72 (1994) 12041207; A. Mohri et al., Jpn. J. Appl. Phys. 37 (1998) L1553-L1555.

[22] H.Boehmer, M. Adams and N. Rynn, Appl. Surf. Science 116 pp23-27 (1997).

[23] R. Bellazini and M.A. Spezziga, INFN PI/AE-94/02.

[24] K. G. Lynn and P. J. Schultz, Review of Modern Physics 60 No 3(1988).

[25] A. P. Mills Jr, The journal of Chemical Physics 62 No 7, 2646-2659 (1975).

[26] Y. Yamazaki, A. Mohri, Y. Kanai, LEAP03 International Conference on Low Energy Antiproton Physics, Yokohama, Japan March 3-7 2003, transparencies presented at leap03, available at http://ad3-proj-leap03.web.cern.ch/ad3-proj-leap03/program.html

[27] M. Yamanaka and Y. Kino, Phys. Rev. A65, 062709 (2002) and references therein.

A. Igarashi, N. Toshima and T. Shirai, J. Phys. B: At. Mol. Opt. Phys. 27, L497-L501 (1994); J. Mitroy and G. Ryzhikh, J. Phys. B: At. Mol. Opt. Phys. 30, L371 (1997); K. Ratnavelu and J. Mitroy, J. Phys. B.,At. Mol. Opt. Phys 28, 287-308 (1995).

[28] J.P. Merrison ,H.Bluhme , M. Charlton , H. Knudsen and M.R. Poulsen, Hyperfine Interactions 109 (1997) 313321.

[29] Chi-Yu Hu, D. Caballero, Z. Hlousek, J. Phys. B: At. Mol. Phys. 34 (2001) 331-338. 
[30] Michael Charlton, Hyperfine Interactions 109 (1997) 269278.

[31] J.P. Merrison et al., Phys. Rev. Lett. 78 (1997) 2728.

[32] P.K. Biswas, J.Phys. B: At. Mol. Opt. Phys. 34 (2001) 4831.

[33] Francis F. Chen, Introduction to Plasma Physics and Controlled Fusion (Plenum, New York, 1983).

[34] Ichioka Toshiyasu, Ph. D Thesis "Development of Intense Beam of Ultra Cold Antiprotons", p84-106.

[35] Yu. L. Sokolov, Determination of the Lamb Shift $(H, n=2)$ by the "Atomic Interferometer" method, 6th International Conference on Atomic Physics Proceedings, August 17-22, 1978, Riga, USSR.

[36] The Hydrogen Atom, Proceedings of the Symposium held in Pisa, Italy, june 30- july 2 1998, Yu. L. Sokolov pp16-29, F. M. Pipkin pp30-38

[37] E. W. Hagley and F. M. Pipkin, Phys. Rev. Lett. 72 No 8, pp1172-1175, 1994.

[38] M.E. Glinsky and T. M. O’Neil, Phys. Fluids B3 (1991) 1279-1293.

[39] F. Robicheaux and J. D. Hanson, Three-body recombination for protons moving in a strong magnetic field, Phys. Rev. A69 (2004) 010701(R); F. Robicheaux, Simulations of antihydrogen formation, Phys. Rev. A70 (2004) 022510.

[40] N. Hoepker, S. Kuzmin and T. O’Neil, private communication. 